Philadelphia College of Osteopathic Medicine DigitalCommons@PCOM

PCOM Scholarly Papers

2016

\title{
A Systematic Review on the Use of Psychosocial Interventions in Conjunction with Medications for the Treatment of Opioid Addiction
}

KDugosh

A. Abraham

B. Seymour

K. McLoyd

M. Chalk

See next page for additional authors

Follow this and additional works at: http://digitalcommons.pcom.edu/scholarly_papers

Part of the Substance Abuse and Addiction Commons

\section{Recommended Citation}

Dugosh, K; Abraham, A.; Seymour, B.; McLoyd, K.; Chalk, M.; and Festinger, David, "A Systematic Review on the Use of Psychosocial Interventions in Conjunction with Medications for the Treatment of Opioid Addiction" (2016). PCOM Scholarly Papers. Paper 1728.

http://digitalcommons.pcom.edu/scholarly_papers/1728

This Article is brought to you for free and open access by DigitalCommons@PCOM. It has been accepted for inclusion in PCOM Scholarly Papers by an authorized administrator of DigitalCommons@PCOM. For more information, please contact library@pcom.edu. 
Authors

K Dugosh, A. Abraham, B. Seymour, K. McLoyd, M. Chalk, and David Festinger 


\title{
A Systematic Review on the Use of Psychosocial Interventions in Conjunction With Medications for the Treatment of Opioid Addiction
}

\author{
Karen Dugosh, PhD, Amanda Abraham, PhD, Brittany Seymour, BA, Keli McLoyd, JD, \\ Mady Chalk, PhD, and David Festinger, PhD
}

\begin{abstract}
Opioid use and overdose rates have risen to epidemic levels in the United States during the past decade. Fortunately, there are effective medications (ie, methadone, buprenorphine, and oral and injectable naltrexone) available for the treatment of opioid addiction. Each of these medications is approved for use in conjunction with psychosocial treatment; however, there is a dearth of empirical research on the optimal psychosocial interventions to use with these medications. In this systematic review, we outline and discuss the findings of 3 prominent prior reviews and 27 recent publications of empirical studies on this topic. The most widely studied psychosocial interventions examined in conjunction with medications for opioid addiction were contingency management and cognitive behavioral therapy, with the majority focusing on methadone treatment. The results generally support the efficacy of providing psychosocial interventions in combination with medications to treat opioid addictions, although the incremental utility varied across studies, outcomes, medications, and interventions. The review highlights significant gaps in the literature and provides areas for future research. Given the enormity of the current opioid problem in the United States, it is critical to gain a better understanding of the most effective ways to deliver psychosocial treatments in conjunction with these medications to improve the health and well-being of individuals suffering from opioid addiction.
\end{abstract}

Key Words: buprenorphine, counseling, medication-assisted treatment, methadone, naltrexone, psychosocial

( $J$ Addict Med 2016;10: 93-103)

From the Treatment Research Institute, Philadelphia, PA (KD, AA, BS, KML, MC, DF); Department of Health Policy and Management, University of Georgia, Athens, GA (AA).

Received for publication July 6, 2015; accepted December 6, 2015.

The study was commissioned by the American Society of Addiction Medicine and partially funded by the National Institute on Drug Abuse.

None of the authors represent any interests that could be interpreted as influential in this research.

Send correspondence and reprint requests to: Karen Leggett Dugosh, Ph.D., Treatment Research Institute, 150 S. Independence Mall West, Suite 600, Philadelphia, PA 19106; E-mail: kdugosh@tresearch.org

Copyright (C) 2016 American Society of Addiction Medicine. This is an openaccess article distributed under the terms of the Creative Commons Attribution-Non Commercial-No Derivatives License 4.0, where it is permissible to download and share the work provided it is properly cited. The work cannot be changed in any way or used commercially.

ISSN: 1932-0620/15/0901-0031

DOI: 10.1097/ADM.0000000000000193
U se of illegal opiates, such as heroin, and the nonmedical use of prescription opioid pain medications, such as oxycodone, have risen to epidemic levels, with rates continuing to soar (Centers for Disease Control and Prevention, 2011). In fact, rates of heroin use in the United States have increased 62\% between 2002 and 2013 (Jones et al., 2015) and an estimated 914,000 individuals have used heroin and 4.3 million individuals used prescription opioids nonmedically in the past year (Center for Behavioral Health Statistics and Quality, 2015). Furthermore, heroin overdose deaths have increased 5-fold from 2001 to 2013 and prescription opioid deaths have increased 3-fold during this same period (National Institute on Drug Abuse, 2015). Although all types of addiction carry risk of harm, opioid addiction is associated with very specific health risks, including transmission of various blood-borne viruses including human immunodeficiency virus (HIV) and hepatitis B and C (Centers for Disease Control and Prevention, 2012). Fortunately, a wide range of treatments including medications and psychosocial interventions are available to manage opioid addiction.

There are currently 3 types of Food and Drug Administration-approved medications to treat opioid addiction: methadone, buprenorphine, and naltrexone (American Society of Addiction Medicine, 2015). Methadone (Methadone [Dolophine] DOLOPHINE [package insert]) is a longacting (24-30 hours) opioid agonist that can be used as both a detoxification medication to suppress withdrawal and cravings and as a maintenance medication to help reduce nonmedical opioid use. Buprenorphine (buprenorphine/ naloxone; (BUNAVAIL [package insert]; SUBOXONE [package insert]; ZUBSOLV [package insert]), buprenorphine monoproduct (SUBUTEX [package insert]), is a partial opioid agonist that, like methadone, can be used during both the detoxification and maintenance stages of treatment for opioid addiction. Finally, naltrexone is a long-acting opioid antagonist that works by binding to opioid receptors for 24 to 30 hours (oral; (DEPADE [package insert]; REVIA [package insert]) or for up to 30 days (extended-release injection; (VIVITROL [package insert]). Because naltrexone blocks opioid receptors, the subjective effects of ingested opioids are significantly reduced, if not completely eliminated. Individuals taking naltrexone must be completely detoxified of all opioids before taking naltrexone, as the interaction will cause immediate opioid withdrawal. As such, naltrexone is not appropriate for use during detoxification, but is particularly 
well suited for maintenance. Results of the systematic reviews (American Society of Addiction Medicine, 2013) have provided unequivocal evidence that, when used as indicated, these medications are both cost effective and clinically effective in reducing opioid use, opioid-related withdrawal and craving, and public health and safety problems related to opioid use (eg, infectious diseases, overdose death, crime).

Each of these medications is approved for use within the framework of medical, social, and psychological support as part of comprehensive treatment for opioid addiction. There is, however, limited research addressing the efficacy of psychosocial interventions used in conjunction with medications to treat opioid addiction. The goal of psychosocial treatment is to help patients control urges to use drugs and remain abstinent, while also serving to assist patients in coping with the emotional strife that often accompanies addiction (Dutra et al., 2008). Psychosocial interventions can be delivered in different treatment modalities (eg, inpatient, outpatient) and in a variety of formats (eg, social skills training, individual, group and couples counseling, cognitive-behavioral therapy [CBT], contingency management (CM), 12-step facilitation therapy, motivational interviewing (MI), family therapy, and others (Carroll and Onken, 2005)]. Although each type of therapy may differ in its structure and theoretical underpinnings, many utilize common therapeutic elements in an aim to (a) modify the underlying processes that serve to maintain addictive behavior, (b) encourage engagement with pharmacotherapy, or (c) treat psychiatric comorbidity that either complicates the addictive disorder or acts as a trigger for relapse. Therefore, selecting a combination of medication and psychosocial treatment that is appropriately targeted and designed to best suit a patient's individual needs is vitally important. The purpose of this paper is to provide a systematic review of the current literature on the use of psychosocial interventions in conjunction with medications for the treatment of opioid addiction.

\section{METHODS}

Procedures for this review generally followed the guidelines set forth by the PRISMA group for systematic reviews (Moher et al., 2009). Articles were identified for inclusion through searches conducted in 2 bibliographic databases (ie, PsycINFO and PubMed) using pre-defined search terms and established selection criteria. In addition, we included in this paper a review of findings from 3 prominent systematic reviews that were conducted on this topic (Drummond and Perryman, 2007; Amato et al., 2011a; Amato et al., 2011b).

\section{Search Criteria}

We identified search terms related to psychosocial treatments, addiction, effectiveness, opioids, and medication (Table 1 for the search terms and strategy). Search terms were intentionally broad to increase the likelihood of identifying all relevant articles. Searches included all fields (eg, titles, abstracts, keywords, etc.) and were restricted to English language and human participants. To increase the overall relevance of the review, we limited the search to articles in the 6-year period from January 1, 2008 to December 31, 2014.

\begin{tabular}{|c|c|}
\hline Search Topic & Search Terms \\
\hline $\begin{array}{r}\text { Psychosocial } \\
\text { treatment }\end{array}$ & $\begin{array}{l}\text { behavioral OR psychosocial OR psychiatric OR } \\
\text { psychological OR twelve step OR } 12 \text { step OR } \\
\text { intervention* OR treatment* OR therap* OR } \\
\text { counsel }^{*} \text { OR psychotherap* }\end{array}$ \\
\hline Addiction & substance use OR substance abuse OR addiction \\
\hline Opioid & opiate $^{*}$ OR opioid* OR heroin OR narcot* \\
\hline Effectiveness & effect ${ }^{*}$ OR efficac* \\
\hline Medication & $\begin{array}{l}\text { medication assisted treatment OR suboxone OR } \\
\text { subutex OR buprenorphine OR methadone OR } \\
\text { naltrexone OR vivitrol }\end{array}$ \\
\hline Final & $\begin{array}{l}\text { Results from Psychosocial Treatment }+ \text { Addiction } \\
+ \text { Opioid }+ \text { Effectiveness }+ \text { Medication }\end{array}$ \\
\hline
\end{tabular}

Filters used on all searches were English, Humans. Date range (January 1, 2008 December 31,2014$)$ was applied on final search (\#13) and the searches were performed on May 16, 2014.

In the event that an article reflected a secondary analysis of data from a relevant study, the original report was included in this review.

\section{Inclusion/Exclusion Criteria}

We included articles describing experimental or quasiexperimental trials examining the efficacy of medication for the treatment of opioid addiction delivered in conjunction with a psychosocial intervention. Articles that were specific to a certain type of a population (eg, pregnant women, adolescents) were also included. Articles that did not include adequate control and whose statistical approach or study design did not allow inference into the efficacy or incremental utility of delivering a psychosocial intervention in combination with medication assisted treatment were excluded as were studies with inadequate sample sizes (ie, less than 15 per group). We also excluded non-empirical articles such as commentaries and editorials. Articles that overlapped in our search and the 3 systemic reviews listed above were not included in our review.

\section{RESULTS}

\section{Search Results}

As seen in Figure 1, the literature searches of the PubMed and PsychINFO databases yielded 793 and 335 results, respectively. Duplicate articles from the 2 searches $(\mathrm{n}=190)$ were removed, resulting in 938 articles for review. The titles and abstracts from these articles were evaluated to determine if they met the inclusion criteria and 151 articles were retained based on this initial screen. These 151 articles were reviewed in their entirety and 27 were identified as suitable for inclusion. Importantly, the team reviewed and reconciled any coding questions (eg, adequacy of control condition) through discussion. These articles along with the relevant systematic reviews of the literature on the use of psychosocial interventions with medications to treat opioid addiction are described in the sections that follow.

\section{Recent Systematic Reviews}

Drummond and Perryman's (2007) review included studies examining the use of psychosocial interventions in 


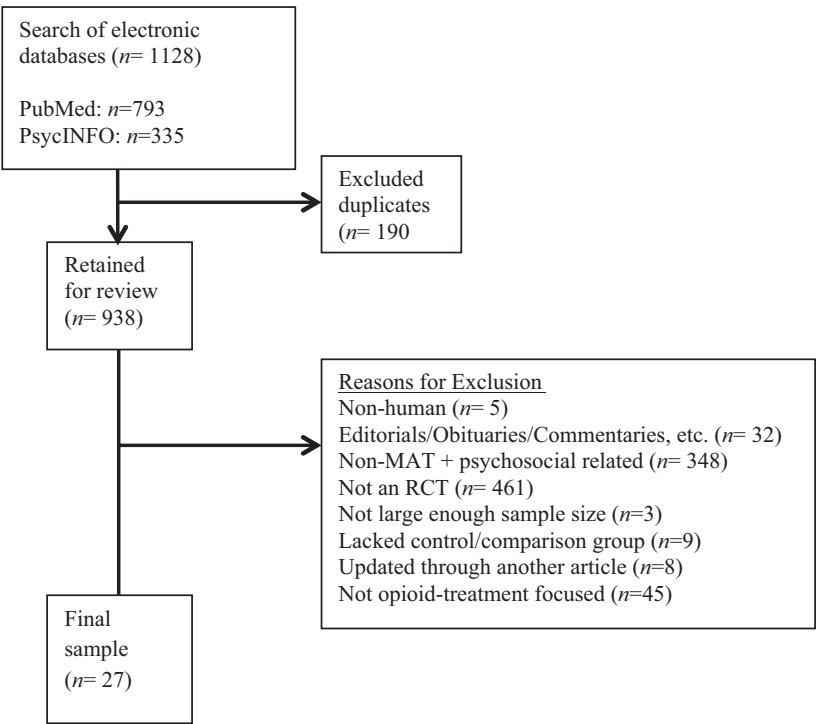

FIGURE 1. Consort diagram of article selection.

conjunction with methadone maintenance therapy (MMT; 8 CBT, 15 CM, 12 standard counseling, 2 family therapy, 8 psychotherapy), buprenorphine treatment (1 CBT, $2 \mathrm{CM}$ ), and naltrexone treatment (1 CM, 1 family therapy, and 1 structured group counseling). Outcomes considered included treatment retention, opioid use, and counseling attendance. There was sufficient evidence to support the use of CM and CBT as an adjunct to MMT, but the authors cautioned that the most effective elements, minimum doses, and active ingredients to observe positive outcomes had not been established. Results also provided some evidence supporting the use of CM in buprenorphine and naltrexone treatment and family therapy in naltrexone treatment. The authors highlighted the need for more and higher quality research to establish the generalizability of the findings to different treatment settings and populations outside of the United States and to examine subgroups for whom different combinations of treatment work best.

In a Cochrane review, Amato et al. (2011a) evaluated the efficacy of providing any psychosocial treatment in conjunction with pharmacological detoxification treatments relative to providing pharmacological detoxification treatments alone. Primary outcomes included program dropout and use of opioids during treatment and at the follow-up assessment(s), and secondary outcomes included clinical absences during the study period, use of other drugs, mortality, and engagement in further treatment. A total of 11 studies were reviewed that encompassed a range of psychosocial interventions (CM, community reinforcement, psychotherapeutic counseling, intensive role inductions (IRIs) with and without case management, counseling and education on high risk behavior, therapeutic alliance intervention, and family therapy) and 2 types of pharmacological detoxification treatments (methadone and buprenorphine). Findings from the systematic review indicated that psychosocial treatments combined with pharmacological detoxification treatments were effective in increasing rates of levels of treatment attendance, improving rates of treatment completion, reducing opioid use, and facilitating longer-term abstinence. Importantly, the available evidence did not provide support for the superiority of any 1 psychosocial approach. The authors cautioned that the findings were limited due to the small number of study participants, heterogeneity of assessments, and lack of detailed outcome information that prevented the possibility of cumulative analysis for several outcomes.

In a second Cochrane review, Amato et al. (2011b) evaluated the efficacy of providing specific psychosocial treatments in conjunction with agonist maintenance treatments. Primary outcomes included treatment retention and abstinence during the study period and at the follow-up assessment(s), and secondary outcomes included the number of psychosocial treatments attended, craving, psychiatric symptoms, quality of life, severity of dependence, and death. A total of 35 studies were reviewed encompassing 13 different psychosocial interventions (ie, acceptance and commitment therapy [ACT], biofeedback, CBT, CM, information-motivation-behavioral skills model, subliminal stimulation, supportive-expressive therapy, short-term interpersonal therapy, customized employment support, enhanced methadone services, enhanced pharmacy services, relational psychotherapies mother's group, and 12-step facilitation) and 3 pharmacological treatments (ie, methadone, buprenorphine, and LAAM). The review concluded that adding any specific psychosocial support to standard maintenance treatments did not add additional benefits for the outcomes considered in the review including treatment retention and opioid use during treatment. These findings were somewhat surprising given that previous versions of the review (Amato et al., 2008) showed a reduction in opioid use during treatment and an increase in the number of participants who were abstinent at the end of the follow-up period.

\section{Psychosocial Interventions and Methadone Maintenance Treatment}

As seen in Table 2, 14 studies evaluated the effectiveness of providing psychosocial treatment in combination with MMT. The interventions examined were CM, CBT, behavioral drug and HIV risk reduction counseling, ACT, general (non-specified) supportive counseling, and a web-based behavioral intervention.

\section{Contingency Management}

Four of the studies examined the efficacy of providing CM in conjunction with MMT, 2 of which were conducted in China (Hser et al., 2011; Chen et al., 2013). Chen et al. (2013) examined the efficacy of prize-based CM in reducing drug use and increasing treatment attendance among MMT patients. Participants were randomly assigned to receive prize-based CM $(=126)$ or MMT $(=120)$ as usual. All participants provided biweekly urine samples for a period of 12 weeks. CM participants earned weekly prize draws on an escalating schedule for providing morphine-negative urine samples and attending MMT. MMT participants received daily methadone dosing and were encouraged to stop using related drugs when they provided a morphine-positive urine sample. Results 
TABLE 2. Articles Included in the Literature Review

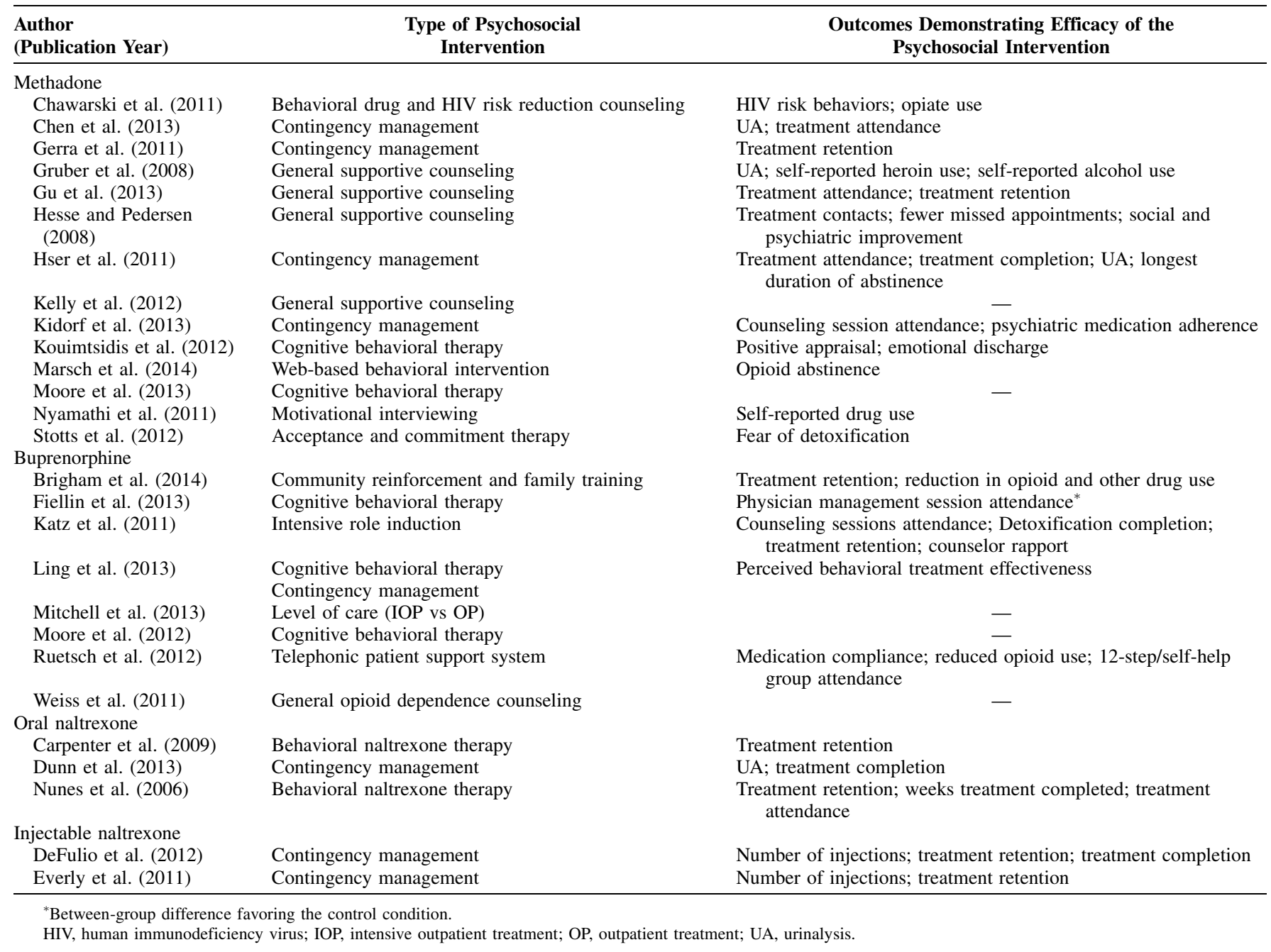

indicated that $\mathrm{CM}$ participants provided more morphine-free urine samples $(P<0.001)$ and attended significantly more days of treatment $(P<0.01)$ than MMT participants.

Hser et al. (2011) randomly assigned MMT clients at 3 sites to receive usual care plus CM $(n=160)$ or MMT $(n=159)$ as usual. MMT participants received a physical examination, weekly urine testing for opioids, and daily supervised methadone doses. In addition to this usual care, CM participants earned prize bowl draws for providing opioid-negative urine specimens and attending MMT on consecutive days. The number of draws earned increased as drug-negative urine provision and consecutive attendance increased. Results showed that CM participants had significantly more weeks of treatment attendance and higher rates of 12 weeks of MMT completion than MMT participants $(P<0.05)$. In addition, CM participants had longer durations of continued abstinence and submitted a significantly greater percentage of urines that were opioid-free than MMT participants $(P<0.05)$.

In a prospective observational multicenter study, Gerra et al. (2011) compared the clinical and psychosocial outcomes of participants in 3 conditions: MMT as usual $(n=100)$, MMT with a contingent take-home program (MMT-C, $\mathrm{n}=100)$, and MMT with a noncontingent take-home program (MMT-NC, $\mathrm{n}=100$ ). MMT participants received supervised daily doses of methadone Monday through Saturday and a take-home dose on Sunday. MMT-C participants visited the center once a week. If they were clinically stable and provided a drug-free urine specimen, they received 7 days of take-home methadone doses. MMT-NC participants received take-home doses after the first week of treatment regardless of their abstinence or other related behaviors. Results showed that MMT-C had a lower risk of treatment dropout than MMT participants $(P=0.02)$. Participants in both the MMT and MMT-C groups showed greater improvement in psychiatric functioning relative to MMT-NC group (both $P$ 's $<0.0001$ ).

Kidorf et al. (2013) compared the efficacy of reinforced on-site integrated care plus vouchers (ROIC, $n=62)$ with standard reinforced on-site integrated care (SOIC, $n=63$ ) in improving outcomes for MMT patients. Participants in both groups received the same scope and schedule of routine drug treatment, which included daily methadone administration 
and an adaptive stepped care counseling approach. The ROIC group received voucher-based incentives (\$25.00/week) for each week in which they attended all of their scheduled psychiatric sessions. ROIC participants attended more counseling sessions in months 1 through 3 of the study than the SOIC group (all $P$ 's $<0.001$ ). Although both groups reported good adherence to psychiatric medications and reductions in psychiatric symptomology over time, ROIC participants demonstrated greater adherence to psychiatric medications in months 1 and 3 of the study ( $P$ 's $=0.02$ and 0.01 , respectively). No differences in substance use outcomes were observed.

\section{Cognitive-behavioral Therapy}

Two studies examined the efficacy of providing CBT in conjunction with MMT relative to providing standard MMT alone. The first study (Kouimtsidis et al., 2012) compared outcomes of participants who were randomly assigned to receive standard MMT $(n=31)$ or standard MMT plus CBT (CBT, $\mathrm{n}=29$ ). MMT participants received 30-minute manual-guided sessions every 2 weeks. In addition to receiving standard MMT, CBT participants were offered weekly individual CBT sessions of 50 minutes for up to 24 sessions during the 6-month study period. Results revealed no significant between-group differences on days of heroin use, abstinence rates, psychosocial problem severity, quality of life, psychological symptoms, or MMT compliance. CBT participants, however, displayed significant improvements in their positive appraisal $(P=0.02)$ at the 6-month assessment and lower emotional discharge at the 12-month assessment $(P<0.05)$ than MMT participants.

The second study (Moore et al., 2013) randomly assigned participants to receive MMT as usual $(n=18)$ or MMT as usual plus Recovery Line (RL, $n=18$ ) for a 4 -week period. RL is a CBT-based therapeutic interactive voice response system designed for MMT patients who continue to use illicit drugs while enrolled in treatment. It incorporates self-monitoring, goal setting, and coping skill rehearsal. Participants in the MMT group received 1 individual psychosocial session per month and were encouraged to attend open access groups that covered a range of topics (eg, methadone, activity scheduling, overdose planning, spirituality). In addition to MMT, RL participants attended an RL orientation session, had 24-hour access to the system for 4 weeks, were given a manual containing information about RL, and received a weekly reminder to use the system. Results indicated no significant differences between the groups in MMT satisfaction, study retention, self-reported substance use, urinalysis-verified opioid and cocaine abstinence, number of counseling sessions attended beyond the minimum requirement, or coping skills. RL participants were, however, more likely to report opioid and cocaine abstinence on days that they used the recovery line relative to days that they did not $(P=0.01)$.

\section{Behavioral Drug and HIV Risk Reduction Counseling}

Chawarski et al. (2011) compared the efficacy of standard MMT plus Behavioral Drug and HIV Risk Reduction
Counseling (BDRC, $\mathrm{n}=20$ ) relative to standard MMT with minimal psychosocial services (MMT, $n=17$ ). Participants assigned to the MMT group could schedule brief visits with a physician, nurse, or counselor as needed to address concerns or crises. Participants assigned to the BDRC group received standard MMT plus weekly, manual-guided BDRC in individual sessions of 45 to 60 minutes. The initial stages of the BDRC intervention focused on the behavioral changes necessary to achieve and maintain abstinence and later stages helped link participant progress in treatment to longer-term recovery goals. The intervention incorporated short-term behavioral contracts and counselors provided feedback and positive reinforcement of participant progress. BDRC individuals had significantly larger reductions in HIV risk behaviors $(P<0.01)$ and opioid use $(P<0.001)$ over time compared with those in the MMT group. Treatment retention rates did not differ significantly between the 2 groups.

\section{Motivational Interviewing}

Nyamathi et al. (2011) examined the relative effectiveness of 3 motivational interviewing (MI) approaches in reducing drug use among MMT clients: MI focused on reduction of drug use delivered in individual sessions (MI$\mathrm{S}, \mathrm{n}=90)$, MI delivered in group sessions (MI-G, $\mathrm{n}=79)$ and a non-MI Nurse-Led Hepatitis Health Promotion $(n=87)$ (Nyamathi et al., 2011). Each program provided 3 sessions to participants and a hepatitis A and B vaccination series for HBV-seronegative participants. Results showed no significant differences among the groups in drug use during the intervention period or at follow-up. The MI-S and MI-G groups both, however, showed significant decreases in self-reported past month drug use at 6-month follow-up $(P=0.0003)$.

\section{Acceptance and Commitment Therapy}

Stotts et al. (2012) randomized participants to receive either drug counseling ( $\mathrm{DC} ; \mathrm{n}=26$ ) or 24-individual therapy sessions of ACT $(n=30)$ as part of a 6-month methadone dose reduction program. Participants in the DC group received 24 weekly 50-minute sessions focusing on abstinent-oriented behaviors and support during the methadone dose reduction period. The ACT group received 24 weekly 50-minute sessions targeting experiential avoidance and fear of the detoxification process in the stabilization period and continuing through the dose reduction period of the study. Although fear of detoxification was reduced over time among participants in the ACT group relative to those in the DC group, there were no significant group differences in treatment attendance or completion, opioid use, treatment success, severity of opioid withdrawal, or engagement in $\mathrm{HIV} / \mathrm{HCV}$ risk behaviors.

\section{General Supportive Counseling}

Four studies examined the efficacy of providing general supportive counseling in conjunction with MMT. The first trial (Gu et al., 2013) compared standard MMT $(n=146)$ to MMT plus a behavioral maintenance therapy-based psychosocial intervention (BMT, $n=142$ ). During their first visit to the clinic, MMT participants received a brief 5 to 15 minute introduction to MMT services and programmatic rules with no other counseling services. The BMT intervention consisted 
of 20 approximately 30-minute counseling sessions delivered by social workers for a 6-month period. The goals of the intervention were to enhance expectation, self-efficacy of maintenance, and satisfaction and experiences related to the particular health-related outcomes and to increase family support and address misconceptions about MMT. Results indicated that BMT participants attended significantly more days of MMT treatment during the study and were less likely to drop out of treatment than MMT participants $(P$ 's $<0.001)$.

In a trial testing effectiveness of MMT among individuals with latent tuberculosis infection, Gruber et al. (2008) randomized participants to 1 of 3 treatment conditions: treatment as usual (TAU, a 21-day methadone detoxification; $\mathrm{n}=39$ ); 6 months of MMT with minimal counseling followed by a 6-week methadone detoxification (MC, $\mathrm{n}=35$ ); or 6 months of MMT with standard counseling followed by a 6week methadone detoxification (SC, $n=37)$. Participants in the $\mathrm{MC}$ group received counseling only on an emergency basis or to enforce program rules, approximately once a month for no more than 15 minutes. Participants in the SC group received counseling twice a month; participants who were employed could earn take-home doses each week that they were drug- and alcohol-free. The 3 groups did not differ significantly in treatment retention, and individuals in the MC and SC groups did not differ significantly on opioid, cocaine, and alcohol use. Relative to those in the TAU condition, individuals in the 2 counseling groups showed a greater decrease in opioid-positive urines and self-reported heroin and alcohol use during the trial (all $P$ 's $<0.05$ ).

A quasiexperimental matched-sample study by Hesse and Pedersen (2008) compared the effectiveness of standard and enhanced psychosocial treatment in improving outcomes of individuals receiving methadone or buprenorphine maintenance treatment. Participants received either standard psychosocial treatment that included case management (SPS, $n=177$ ) or an enhanced psychosocial treatment that included case management, access to a drop-in center, and access to staff members (EPS, $n=126)$. Results indicated that participants in the EPS group had significantly more treatment contacts $(P=0.04)$ and fewer missed appointments $(P<0.0001)$ than those in the SPS group. Social and psychiatric improvement was higher among EPS participants ( $P$ 's $<0.05$ and 0.01 , respectively); conversely, financial improvement was higher among SPS participants $(P<0.05)$. The groups did not differ on self-reported drug or alcohol use.

The fourth study (Kelly et al., 2012) examined the efficacy of different interventions designed to reduce HIV risk among MMT participants. Individuals were randomly assigned to receive 1 of 3 interventions: Interim Methadone (IM, $n=99)$, Standard Methadone (SM, $\mathrm{n}=104)$, or Restored Methadone $(\mathrm{RS}, \mathrm{n}=27)$. IM participants received up to 120 days of administered methadone with only emergency counseling and no take-home doses of methadone. SM participants received methadone and standard counseling (ie, weekly individual sessions for the first 30-60 days followed by biweekly or monthly sessions as needed). They were eligible to earn takehome doses for adhering to treatment requirements and providing drug-negative urines. RM participants received methadone and DC on an as-needed basis determined by the individual or the counselor; they were also eligible to earn take-home doses. For the purposes of the analyses, the SM and RM groups were combined and evaluated relative to the IM group. Results did not support the efficacy of the enhanced interventions in reducing HIV risk as there were no significant differences between the groups in HIV-related outcomes. This study did not evaluate outcomes related to opioid use.

\section{Web-based Behavioral Interventions}

Marsch et al. (2014) examined the efficacy of a webbased behavioral intervention used in conjunction with MMT. Study participants were randomly assigned to receive standard adjunctive drug treatment (TAU, $\mathrm{n}=80$ ) or reduced standard treatment plus the Therapeutic Education System (TES, $\mathrm{n}=80$ ). TES is an interactive, self-directed, web-based tool consisting of 65 modules that address a broad range of skills and behaviors related to successful cessation of substance use and life skills including cognitive-behavioral and relapse prevention skills training and HIV prevention. TAU participants received 1 hour weekly counseling sessions focusing on rehabilitation and program compliance in the first month of treatment and biweekly thereafter. Counselors offered limited case management to participants and participants could receive HIV educational materials. Participants in the TES group received the same counseling offered to the standard treatment group with the exception that each participant was asked to spend half of each scheduled counseling session with their counselor (30 minutes) and the other half using TES (30 minutes). TES participants were more likely to be abstinent TAU participants $(P<0.05)$. The 2 groups did not differ in treatment retention.

\section{Methadone Maintenance Therapy Summary}

In general, the studies reviewed provide support for the use of psychosocial interventions in the context of MMT. Nine of the 14 studies reviewed reported significant effects of the psychosocial treatment on treatment attendance and drug use. Specifically, 5 studies (Hesse and Pedersen, 2008; Hser et al., 2011; Chen et al., 2013; Gu et al., 2013; Kidorf et al., 2013) demonstrated greater treatment attendance and 2 studies (Gerra et al., 2011; Gu et al., 2013) demonstrated lower treatment dropout rates when psychosocial treatment was provided relative to a comparison group. Five studies (Gruber et al., 2008; Chawarski et al., 2011; Hser et al., 2011; Chen et al., 2013; Marsch et al., 2014) demonstrated decreased opioid use among MMT clients receiving psychosocial treatment relative to a comparison group. In addition, 7 studies revealed significant effects of psychosocial interventions on secondary outcomes including HIV risk (Chawarski et al., 2011), psychosocial functioning (Hesse and Pedersen, 2008; Gerra et al., 2011), adherence to psychiatric medications (Kidorf et al., 2013), alcohol use (Gruber et al., 2008), and fear of detoxification (Stotts et al., 2012) relative to a comparison group. It should be noted that the comparison groups varied across studies and the majority were not MMT-only conditions.

\section{Psychosocial Interventions Delivered in Conjunction With Buprenorphine Treatment}

Eight studies were identified that examined the efficacy of delivering psychosocial treatment within the context of 
buprenorphine treatment. Psychosocial treatments examined included CBT $(n=3)$, community reinforcement and family training (CRAFT-T) $(n=1)$, IRI $(n=1)$, general supportive counseling $(\mathrm{n}=1)$, and a telephonic patient support system $(\mathrm{n}=1)$. In addition, 1 study examined the delivery of psychosocial counseling within different levels of care $(n=1)$.

\section{Cognitive-behavioral Therapy}

Three studies examined the use of CBT in the context of buprenorphine treatment (Moore et al., 2012; Fiellin et al., 2013; Ling et al., 2013). The first (Fiellin et al., 2013) compared the efficacy of providing physician management alone $(\mathrm{PM}=71)$ to $\mathrm{PM}$ plus $\mathrm{CBT}(\mathrm{PM}+\mathrm{CBT}=70)$ in a primary care setting. Physician management included up to 8 sessions lasting approximately 15 to 20 minutes. In the session, an internist reviewed the patient's recent drug use, provided brief advice on achieving or maintaining abstinence, reviewed medical and psychiatric symptoms, assessed social, work, and legal function, reviewed selfhelp attendance, and discussed weekly urine toxicology results. Participants in the PM+CBT group were also offered up to twelve 50-minute weekly manualized CBT sessions (delivered by a master's or doctoral-level clinician) during the first 12 weeks of treatment. There were no significant differences between the 2 groups in opioid use and study completion; however, both groups did report a reduction in self-reported frequency of opioid use $(P<0.001)$. In addition, PM participants attended more physician management sessions than PM+CBT participants $(P<0.01)$.

A similar study by Moore et al. (2012) compared PM with weekly buprenorphine dispensing (PM, $n=28)$ with PM plus directly observed buprenorphine consumption and CBT (CBT, $\mathrm{n}=27)$. The study found no significant differences between the groups in treatment retention, maximum number of consecutive weeks of opioid abstinence, or participant satisfaction. Among individuals in the CBT group, CBT session attendance rate was positively associated with opioid-negative urine provision $(P=0.05)$ and maximum continuous weeks of opioid abstinence $(P=0.007)$.

A third study by Ling et al. (2013) examined the efficacy of both CBT and CM in improving outcomes for individuals receiving buprenorphine treatment. Participants were assigned to 1 of 4 groups: CBT $(n=53)$; CM $(n=49)$; $\mathrm{CBT}+\mathrm{CM}(\mathrm{n}=49)$; or no additional psychosocial treatment $(\mathrm{NT}, \mathrm{n}=51)$. Participants receiving CBT met with a master's level trained counselor for weekly 45-minute individual sessions to address topics relevant to recovery and completed related exercises and homework. Participants receiving CM could earn prize draws for providing drug-negative urines on a weekly basis using an escalating schedule. Although all groups showed a significant reduction in opioid use from baseline to the end of the treatment phase, there were no significant between-group differences on treatment retention, withdrawal and craving, psychosocial problem severity, or medication and treatment compliance. Treatment satisfaction was very high across the 4 groups $(71 \%-81 \%$ very satisfied with their treatment); however, participants in the NT group were significantly more likely to report that their behavioral treatment was "not effective" compared with the other 3 groups $(P=0.007)$.

\section{Community Reinforcement and Family Training for Treatment Retention}

One study (Brigham et al., 2014) evaluated the efficacy of using community reinforcement and family training for treatment retention (CRAFT-T) in the context of buprenorphine treatment. This intervention is designed to work with identified participants (IPs) and concerned significant others (CSOs) who are already engaged in treatment to increase treatment retention and recovery support. In this study, opioid-dependent adults (IPs) who were enrolled in a buprenorphine-detoxification program and their identified CSO were randomly assigned to receive the CRAFT-T intervention $(\mathrm{n}=28$ dyads) or TAU ( $n=24$ dyads). In the CRAFT-T intervention, the IP and CSO met with a therapist for 2 joint sessions and the CSO met individually with the therapist for 10 sessions. The primary outcome was the number of days to IP dropout from treatment and the secondary outcomes were days of IP opioid use and any other drug use. Results showed that CRAFT-T participants with parental family CSOs had greater treatment retention than those with a nonparental family CSO, TAU participants with a parental-family CSO, or TAU participants with a nonparental family CSO $(P<0.01)$. IPs assigned to the CRAFT-T group showed significantly greater reductions in opioid and other drug use (both $P$ 's $<0.0001$ ).

\section{Intensive Role Induction}

A study by Katz et al. (2011) randomized participants to buprenorphine detoxification with standard treatment (ST, $\mathrm{n}=83$ ), IRI $(\mathrm{n}=81)$, or IRI plus case management (IRI+CM, $\mathrm{CM}, \mathrm{n}=76$ ). ST participants received individual counseling sessions over the 30-day detoxification period and 5 weekly counseling sessions. Sessions followed the disease model of addiction and addressed participants' issues and concerns. IRI participants attended 5 weekly IRI counseling sessions to enhance treatment engagement and provide psychoeducation about detoxification. In addition to IRI, participants in the IRI+CM group received assistance from their counselor to access community resources to support recovery. Results showed that individuals in the IRI and IRI+CM group attended significantly more counseling sessions during detoxification than the ST group $(P<0.001)$ and those in the IRI group were more likely to complete detoxification than those in the ST group $(P=0.017)$. In addition, IRI participants were retained in treatment for more days after detoxification than ST participants $(P=0.005)$, and IRI participants rated their counselor more positively at 1-month follow-up than ST participants $(P=0.01)$. There were no significant differences between the IRI+CM group and ST group on these 3 outcomes.

\section{General Opioid Dependence Counseling}

One study (Weiss et al., 2011) examined the effectiveness of a manualized opioid dependence counseling intervention used in conjunction with buprenorphine during Phase 1 (brief treatment: buprenorphine-naloxone induction, 2 weeks of stabilization, 2 week taper, and 8 weeks of follow-up) and Phase 2 (extended treatment: 12 weeks of buprenorphinenaloxone stabilization, 4 week taper, and 8 weeks of followup). Participants were randomly assigned to a manual-based medical management delivered by a buprenorphine-certified 
physician (MM, $\mathrm{n}=180)$ or SMM plus opioid dependence counseling $(n=180)$ group. The initial visit in each phase of the study was 45 to 60 minutes and subsequent visits were 15 to 20 minutes. In addition to SMM, participants in the opioid dependence counseling group received a manual-based opioid dependence counseling delivered in 45 to 60 minutes sessions by a trained addictions or mental health professional. The counseling incorporated an interactive skill-based format with takehome assignments to address addiction and recovery, recommend self-help groups, and emphasize lifestyle changes. Results indicated no significant differences between the 2 groups in session attendance, medication dose and protocol adherence, attendance at SMM visits, or opioid use outcomes.

\section{Telephonic Participant Support System}

Ruetsch et al. (2012) randomized individuals to receive standard buprenorphine care (TAU, $n=439)$ or to the HereToHelp (HTH) intervention $(\mathrm{n}=987)$, a telephonic participant support system. HTH participants received coaching calls (2-3 times per month for a total of 8 calls) providing education about overdose and treatment, resolving challenges within treatment, and encouraging individuals to stay in treatment. The study found that compliance with buprenorphine was greater in the HTH group than the TAU group for participants with 3 or more, 4 or more, 5 or more, or 7 or more sessions (all $P$ 's $<0.05$ ). Participants who completed a greater number of calls were also more likely to be compliant with buprenorphine at month $12(P<0.001)$. Participants in the HTH group were significantly less likely to use opioids at month $12(P<0.05)$ and significantly more likely to attend 12 -step/self-help groups $(P<0.05)$ than those in the standard care group. There were no significant between-group differences on addiction severity index (ASI) composite scores.

\section{Psychosocial Counseling Within Different Levels of Care}

A recent study compared the outcomes of buprenorphine treatment clients who were randomly assigned to receive intensive outpatient treatment $(\mathrm{OP}, \mathrm{n}=145)$ and $\mathrm{OP}$ $(\mathrm{n}=155)$ (Mitchell et al., 2013). Intensive outpatient treatment participants attended at least 9 hours of counseling per week for about 45 days $(4 \mathrm{~d} / \mathrm{wk}$ for at least $2 \mathrm{~h} / \mathrm{d}$, plus 1 individual session) and OP participants attended a minimum of 1 group session and 1 individual counseling session per week (up to 8 hours of counseling per week). Sessions typically focused on substance use education, relapse prevention, medication education, HIV prevention, health promotion, and women's support groups; 12-step attendance was encouraged. Although both groups showed statistically significant improvements on 16 outcomes, there were no significant between-group differences for the 20 outcomes considered in the study (eg, opioid-negative urines, days of heroin use, days of crime, treatment retention, ASI composite, quality of life, HIV risk behaviors).

\section{Buprenorphine Treatment Summary}

In general, the support for the efficacy of delivering concurrent psychosocial interventions was less robust for buprenorphine. Three of the 8 studies reviewed found significant effects of the psychosocial treatment on treatment attendance and drug use. One study (Katz et al., 2011) demonstrated higher rates of treatment retention, completion, and attendance among groups receiving concurrent psychosocial treatment. Two studies (Brigham et al., 2014) found reductions in opioid use in groups assigned to receive psychosocial interventions, and 1 study (Ruetsch et al., 2012) found that it improved buprenorphine compliance. In addition, 3 studies found significant differences for secondary outcomes including treatment satisfaction (Ling et al., 2013), counselor rating (Katz et al., 2011; Ruetsch et al., 2012), and 12-step/self-help meeting attendance (Ruetsch et al., 2012).

\section{Psychosocial Interventions Delivered in Conjunction With Oral Naltrexone}

Only 3 studies (one of which was a secondary analysis) examined the efficacy of providing psychosocial treatment concurrently with oral naltrexone treatment (Nunes et al., 2006; Carpenter et al., 2009; Dunn et al., 2013). In 1 study (Dunn et al., 2013), all participants were invited to attend a therapeutic workplace for 26 weeks and were eligible to receive voucher payments for participation in the workplace. Individuals were randomly assigned to either a noncontingent group $(\mathrm{NC}, \mathrm{n}=32)$ in which they were allowed free access to the workplace or a contingent group $(\mathrm{CM}, \mathrm{n}=35)$ in which they were required to ingest oral naltrexone under supervision to gain access. Results showed that CM participants provided more naltrexone-positive urine samples at the 30-day assessment $(P<0.01)$, were more likely to provide $100 \%$ naltrexone-positive urine samples $(P<0.01)$, and were more likely to complete naltrexone treatment $(P<0.01)$ compared with NC participants. In addition, CM participants had significantly more opioid-negative urine samples than NC participants when missing data were not imputed as positive $(P=0.01)$. These findings are similar to the findings of 2 studies of extended-release injectable naltrexone with psychosocial treatment described in the next section (Everly et al., 2011; DeFulio et al., 2012).

One study and secondary analysis examined the differences between oral naltrexone participants receiving compliance enhancement (CE) and behavioral naltrexone therapy (BNT). CE is a manual-guided psychoeducational intervention designed to be an adequate control condition for medication trials that emphasizes medication adherence and includes discussions of naltrexone compliance, supportive problem-solving, and 12-step principles. BNT is a manualguided intervention combining elements of MI, Relapse Prevention Therapy, Network Therapy, and CM to increase adherence to oral naltrexone and reduce relapse to opioids. Nunes et al. (2006) found that BNT participants $(n=36)$ were more likely to be retained in treatment for the 6-month study period $(P=0.04)$ than participants in the CE group $(\mathrm{n}=33)$. They also completed more weeks of treatment $(P=0.04)$ and had a higher proportion of sessions attended $(P=0.03)$. A secondary analysis of this data (Carpenter et al., 2009) demonstrated that the transition rate from abstinence to treatment dropout was approximately 3.5 times greater among participants in the CE group than the BNT group $(P<0.05)$. 


\section{Oral Naltrexone Summary}

The 3 studies of the use of psychosocial treatment in combination with oral naltrexone demonstrated positive effects of the psychosocial intervention on treatment retention and attendance (Nunes et al., 2006; Dunn et al., 2013) and oral naltrexone compliance (Dunn et al., 2013). Again, it should be noted that the comparison group varied across studies and the majority were not comprised of medications alone.

\section{Psychosocial Interventions Delivered in Conjunction With Extended-release Injectable Naltrexone}

Two studies (Everly et al., 2011; DeFulio et al., 2012) were identified that examined the use of psychosocial treatment in conjunction with injectable naltrexone to treat opioid addiction. Both studies employed the methods of Dunn et al. (2013) described above to examine the efficacy of providing $\mathrm{CM}$ in the context of a therapeutic workplace to individuals receiving injectable naltrexone. In each of the studies, individuals in the $\mathrm{NC}(\mathrm{n}=17, \mathrm{n}=19$, respectively) were offered injectable naltrexone injections and were allowed to access the workplace independent of whether the injections were accepted. Individuals in the $\mathrm{CM}(\mathrm{n}=18, \mathrm{n}=19$, respectively) were required to accept the injections to gain and maintain access to the therapeutic workplace. Results of both studies showed that $\mathrm{CM}$ individuals received more injections and had higher rates of retention than $\mathrm{NC}$ individuals $(P=0.026$, $P=0.008$, respectively). In addition, DeFulio et al. (2012) found that a greater percentage of CM participants completed the entire 24 weeks of injectable naltrexone than NC participants $(P=0.004)$. In both studies, there were no differences in retention in the therapeutic workplace, urinalysis-verified opioid use, or voucher earnings between the 2 groups.

\section{Injectable Naltrexone Summary}

The 2 studies (Everly et al., 2011; DeFulio et al., 2012) examining the use of psychosocial treatments in conjunction with injectable naltrexone demonstrated the efficacy of providing $\mathrm{CM}$ in increasing the number of injections received and treatment retention. In addition, DeFulio et al. (2012) found higher completion rates for the 24-week course of injectable naltrexone. These studies, however, revealed no significant differences in opioid use.

\section{DISCUSSION}

Despite the robust body of research conducted for several decades on the use of medication-assisted treatment for the treatment of opioid addiction, few physicians outside of the addiction specialty field are familiar with these medications despite their robust treatment effects. Given the current state of the opioid overdose epidemic, it is critical that patients seeking help for opioid addiction have access to comprehensive treatment that includes these highly effective medications whose effects may be enhance with the provision of psychosocial interventions. A clear understanding of the safest and most effective combination of the 2 is imperative to successfully treat opioid addiction.

This literature review represents the current body of knowledge regarding medications for the treatment of opioid addiction in conjunction with psychosocial treatment. Results from the reviewed studies generally support the efficacy of providing psychosocial therapy in combination with medications for the treatment of opioid addiction in improving clinical outcomes. The incremental efficacy of adding psychosocial interventions to medically assisted treatment, however, varied for different outcomes, across studies, and within psychosocial intervention types. This can likely be attributed to the fact that the comparison groups were not consistent across studies. In most cases, control groups did not consist of providing medication alone, and medication management control group conditions may have been more intensive than would be encountered in clinical practice (eg, involved more frequent physician contact and/or longer duration of visits). It is possible that the effects would have been stronger and more consistent if the comparison conditions provided medication alone or reflected the level of medication management that currently occurs in clinical practice.

Perhaps the most striking aspect of the research seems to be the lack of information about the use of specific medications in combination with specific types of psychosocial interventions during all phases of treatment and among different subpopulations. The importance of developing best practices and clinical guidelines in this area is essential. With the rising number of Americans who are struggling with opioid addiction, and with the passage of the Affordable Care and Parity Acts, it is likely that there will be an increase in patient demand for opioid addiction treatment from their general and primary care physicians. It is imperative to ensure that the physicians understand that the best outcomes for patients taking these medications may be achieved when they are used in conjunction with psychosocial interventions. Furthermore, it is essential to build a body of research knowledge that can guide practitioners in determining the most appropriate medication/psychosocial intervention combinations for individual patients.

This systematic review has several limitations. First, it is possible that studies were excluded because we did not include unpublished research findings or papers published in languages other than English and we did not perform extensive cross-referencing of the literature cited in identified papers. Second, the findings of the current review may be tempered because we did not include a formal assessment of risk of bias. We, however, attempted to overcome this limitation by including only studies with adequate scientific rigor by eliminating those that had no or insufficient control groups, did not allow inference into the incremental utility of the psychosocial intervention, or had inadequate sample sizes.

\section{CONCLUSIONS}

This literature review highlights significant gaps in the research base regarding use of psychosocial interventions in conjunction with medications for the treatment of opioid addiction. Although many studies have examined psychosocial treatment as an adjunct to methadone maintenance therapy, relatively fewer studies have examined it as an adjunction to buprenorphine or naltrexone treatment, which can be delivered in primary care settings. For example, this review revealed only 5 studies of psychosocial treatment as an 
adjunct to naltrexone treatment. Although naltrexone is not appropriate for use in detoxification, it is a viable option for the treatment of opioid addiction in motivated individuals who are abstinent from opioids and, unlike methadone and buprenorphine, naltrexone can be prescribed by any licensed physician or other medical professionals with prescribing authority. Furthermore, there is little empirical evidence suggesting which psychosocial treatments work best in conjunction with medication-assisted treatment as there are relatively few studies comparing the differential effectiveness of various psychosocial approaches (eg, CM, MI) for individuals receiving medications for the treatment of opioid addiction.

There are many important areas for future research that should be explored immediately, given the enormity of the opioid problem and evidence suggesting that psychosocial interventions can be an important part of comprehensive, recovery-oriented treatment. Examples of topics that should be explored further include whether specific forms of psychosocial interventions are most effective in combination with specific types of medications, whether different approaches work better at different points along the treatment continuum, and what psychosocial interventions delivered with medications are most effective for different patient populations and treatment settings including primary care. As opioid use and overdose deaths in this country exceed epidemic proportions, the urgency for an expanded research agenda on best practices for comprehensive treatment could not be more critical.

\section{REFERENCES}

Amato L, Minozzi S, Davoli M, et al. Psychosocial and pharmacological treatments versus pharmacological treatments for opioid detoxification. Cochrane Database Syst Rev 2008;(4). CD005031.

Amato L, Minozzi S, Davoli M, et al. Psychosocial and pharmacological treatments versus pharmacological treatments for opioid detoxification. Cochrane Database Syst Rev 2011;(9). CD005031.

Amato L, Minozzi S, Davoli M, et al. Psychosocial combined with agonist maintenance treatments versus agonist maintenance treatments alone for treatment of opioid dependence. Cochrane Database Syst Rev 2011;(10). CD004147.

American Society of Addiction Medicine. Advancing Access to Addiction Medicine: Implications for Opioid Addiction Treatment. American Society of Addiction Medicine; 2013.

American Society of Addiction Medicine. The ASAM National Practice Guideline for the Use of Medications in the Treatment of Addiction Involving Opioid Use. American Society of Addiction Medicine; 2015.

Brigham GS, Slesnick N, Winhusen TM, et al. A randomized pilot clinical trial to evaluate the efficacy of community reinforcement and family training for treatment retention (CRAFT-T) for improving outcomes for patients completing opioid detoxification. Drug Alcohol Depend 2014;138:240-243.

BUNAVAIL [package insert]. Raleigh, NC: BioDelivery Sciences International, Inc.; Last Revised September; 2014.

Carpenter KM, Jiang H, Sullivan MA, et al. Betting on change: modeling transitional probabilities to guide therapy development for opioid dependence. Psychol Addict Behav 2009;23(1):47-55.

Carroll KM, Onken LS. Behavioral therapies for drug abuse. Am J Psychiatry 2005;162(8):1452-1460.

Center for Behavioral Health Statistics and Quality. Behavioral Health Trends in the United States: Results From the 2014 National Survey on Drug Use and Health (HHS Publication No. SMA 15-4927, NSDUH Series H-50).
Center for Behavioral Health Statistics and Quality; 2015, http:// www.samhsa.gov/data/. Accessed October 15, 2015.

Centers for Disease Control and Prevention. Vital sign: overdoses of prescription opioid pain relievers-United States, 1999-2008. MMWR Morb Mortal Wkly Rep 2011;60(43):1487-1492. http://www.cdc.gov/mmwr/ preview/mmwrhtml/mm6043a4.htm. Accessed October 15, 2015.

Centers for Disease Control and Prevention. New HIV infections in the United States. Centers for Disease Control and Prevention; 2012.

Chawarski MC, Zhou W, Schottenfeld RS. Behavioral drug and HIV risk reduction counseling (BDRC) in MMT programs in wuhan, china: A pilot randomized clinical trial. Drug Alcohol Depend 2011;115(3):237-239.

Chen W, Hong Y, Zou X, et al. Effectiveness of prize-based contingency management in a methadone maintenance program in china. Drug Alcohol Depend 2013;133(1):270-274.

DeFulio A, Everly JJ, Leoutsakos J-MS, et al. Employment-based reinforcement of adherence to an FDA approved extended release formulation of naltrexone in opioid-dependent adults: a randomized controlled trial. Drug Alcohol Depend 2012;120(1-3):48-54.

DEPADE [package insert]. Hazelwood, MO: Mallinckrodt, Inc.; Last Revised November 2014.

Drummond DC, Perryman K. Psychosocial Interventions in Pharmacotherapy of Opioid Dependence: A Literature Review. London: St. George's University of London; 2007.

Dunn KE, Defulio A, Everly JJ, et al. Employment-based reinforcement of adherence to oral naltrexone treatment in unemployed injection drug users. Exp Clin Psychopharmacol 2013;21(1):74-83.

Dutra L, Stathopoulou G, Basden SL, et al. A meta-analytic review of psychosocial interventions for substance use disorders. Am J Psychiatry 2008;165(2):179-187.

Everly JJ, DeFulio A, Koffarnus MN, et al. Employment-based reinforcement of adherence to depot naltrexone in unemployed opioid-dependent adults: a randomized controlled trial. Addiction 2011;106(7):1309-1318.

Fiellin DA, Barry DT, Sullivan LE, et al. A randomized trial of cognitive behavioral therapy in primary care-based buprenorphine. Am J Med 2013;126(1):74.e11-77.e11.

Gerra G, Saenz E, Busse A, et al. Supervised daily consumption, contingent take-home incentive and non-contingent take-home in methadone maintenance. Prog Neuropsychopharmacol Biol Psychiatry 2011;35(2):483489.

Gruber VA, Delucchi KL, Kielstein A, et al. A randomized trial of 6-month methadone maintenance with standard or minimal counseling versus 21day methadone detoxification. Drug Alcohol Depend 2008;94(1-3):199206.

$\mathrm{Gu}$ J, Lau JT, Xu H, et al. A randomized controlled trial to evaluate the relative efficacy of the addition of a psycho-social intervention to standard-of-care services in reducing attrition and improving attendance among first-time users of methadone maintenance treatment in China. AIDS Behav 2013;17(6):2002-2010.

Hesse M, Pedersen MU. Easy-access services in low-threshold opiate agonist maintenance. Int J Ment Health Addict 2008;6(3):316-324.

Hser YI, Li J, Jiang H, et al. Effects of a randomized contingency management intervention on opiate abstinence and retention in methadone maintenance treatment in China. Addiction 2011;106(10):1801-1809.

Jones CM, Logan J, Gladden RW, et al. Vital signs: demographic and substance use trends among heroin users: United states, 2002-2013. MMWR Morb Mortal Wkly Rep 2015;64(26):719-725.

Katz EC, Brown BS, Schwartz RP, et al. Transitioning opioid-dependent patients from detoxification to long-term treatment: efficacy of intensive role induction. Drug Alcohol Depend 2011;117:24-30.

Kelly SM, Schwartz RP, O'Grady KE, et al. Impact of methadone with versus without drug abuse counseling on HIV risk: 4- and 12-month findings from a clinical trial. J Addict Med 2012;6(2):145-152.

Kidorf M, Brooner RK, Gandotra N, et al. Reinforcing integrated psychiatric service attendance in an opioid-agonist program: a randomized and controlled trial. Drug Alcohol Depend 2013;133(1):30-36.

Kouimtsidis C, Reynolds M, Coulton S, et al. How does cognitive behaviour therapy work with opioid-dependent clients? Results of the UKCBTMM study. Drugs: Educ Prev Polic 2012;19(3):253-258.

Ling W, Hillhouse M, Ang A, et al. Comparison of behavioral treatment conditions in buprenorphine maintenance. Addiction 2013;108(10): 17881798. 
Marsch LA, Guarino H, Acosta M, et al. Web-based behavioral treatment for substance use disorders as a partial replacement of standard methadone maintenance treatment. $J$ Subst Abuse Treat 2014;46(1):43-51.

Methadone (Dolophine ${ }^{\circledR}$ ) DOLOPHINE [package insert]. Columbus, $\mathrm{OH}$ : Roxane Laboratories, Inc.; Revised April 2015.

Mitchell SG, Gryczynski J, Schwartz RP, et al. A randomized trial of intensive outpatient (IOP) vs. standard outpatient (op) buprenorphine treatment for African Americans. Drug Alcohol Depend 2013;128(3):222-229.

Moher D, Liberati A, Tetzlaff J, et al. Preferred reporting items for systematic reviews and meta-analyses: the prisma statement. Ann Intern Med 2009;151(4):264-269.

Moore BA, Barry DT, Sullivan LE, et al. Counseling and directly observed medication for primary care buprenorphine maintenance: a pilot study. $J$ Addict Med 2012;6(3):205-211.

Moore BA, Fazzino T, Barry DT, et al. The recovery line: a pilot trial of automated, telephone-based treatment for continued drug use in methadone maintenance. J Subst Abuse Treat 2013;45(1):63-69.

National Institute on Drug Abuse. Overdose death rates. 2015. https:// www.drugabuse.gov/related-topics/trends-statistics/overdose-death-rates. Accessed October 15, 2015.

Nunes EV, Rothenberg J, Sullivan MA, et al. Behavioral therapy to augment oral naltrexone for opioid dependence: a ceiling on effectiveness. Am J Drug Alcohol Abuse 2006;32:503-517.
Nyamathi AM, Nandy K, Greengold B, et al. Effectiveness of intervention on improvement of drug use among methadone maintained adults. $J$ Addict Dis 2011;30(1):6-16.

REVIA [package insert]. Pomona, NY: Barr Pharmaceuticals, Inc.; Revised December 2013.

Ruetsch C, Tkacz J, McPherson TL, et al. The effect of telephonic patient support on treatment for opioid dependence: outcomes at one year followup. Addict Behav 2012;37(5):686-689.

Stotts AL, Green C, Masuda A, et al. A stage I pilot study of acceptance and commitment therapy for methadone detoxification. Drug Alcohol Depend 2012;125(3):215-222.

SUBOXONE [package insert]. Richmond, VA: Reckitt Benckiser Pharmaceuticals Inc.; Last Revised April 2014.

SUBUTEX [package insert]. Montgomery, AL: Midlothian Laboratories; Last Revised September 2010.

VIVITROL [package insert]. Waltham, MA: Alkermes, Inc.; Last Revised July 2013.

Weiss RD, Potter JS, Fiellin DA, et al. Adjunctive counseling during brief and extended buprenorphine-naloxone treatment for prescription opioid dependence: a 2-phase randomized controlled trial. Arch Gen Psychiatry 2011;68(12):1238-1246.

ZUBSOLV [package insert]. Morristown, NJ: Orexo US, Inc.; Last Revised December 2014. 